\title{
Allergen Immunotherapy (AIT) in Allergic Rhinitis: Long-Term Efficacy and the Development of Asthma. What Do We Know?
}

Carmen Vidal, $M D, P h D^{1, *}$

Pablo Rodríguez del Río, $M D^{2}$

Ana Tabar, PhD

Carmen Moreno, MD, $P h D^{4}$

\author{
Address \\ 1, Allergy Department, Complejo Hospitalario Universitario de Santiago, Rúa \\ Ramón Baltar sn, 15706 Santiago de Compostela, Spain \\ ${ }^{2}$ Allergy Department, Hospital del Niño Jesús, Avda. Menéndez Pelayo 65, \\ 28009 Madrid, Spain \\ ${ }^{3}$ Allergy Department, Complejo Hospitalario de Navarra, Plaza de la Paz sn, \\ 31002 Pamplona, Spain \\ ${ }^{4}$ Allergy Department, Hospital Reina Sofía, Avda. Menéndez Pidal sn, 14004 \\ Córdoba, Spain \\ Published online: 14 January 2014 \\ (C) Springer International Publishing AG 2014
}

Keywords Allergic rhinitis - Asthma - Allergen - Immunotherapy - Long-term efficacy - Prevention - Outcomes . Clinical trial

\section{Opinion statement}

Allergy Immunotherapy is the only treatment able to modify the natural history of the respiratory allergic disease. It is indicated in IgE-mediated rhinoconjunctivitis and/or allergic asthma in both children and adults whenever more than occasional use of antiinflammatory or controller medications is needed. Only well-standardized products should be used after precise diagnosis has been achieved. Efficacy of allergen immunotherapy (AIT) with mite and pollen extracts persist once treatment is discontinued, and for that AIT may be considered the only treatment able to change the natural history of the allergic disease.

\section{Key points}

1. The strongest risk for developing asthma occurred in those patients with rhinitis and sensitization to several inhalant allergens, particularly house dust mites.

2. The role of allergen avoidance measures in the evolution of the respiratory disease is unknown. 
3. AIT is indicated for rhinoconjunctivitis and/or asthma due to allergy to inhalants. In both cases, it is preferable to initiate the treatment as soon as possible, before chronic irreversible changes occur.

4. According to the European Medicines Agency (EMA), long-term efficacy can be defined as "the prolonged clinically relevant benefit that persists after stopping the treatment".

5. Long-term efficacy of AIT has been demonstrated for both mite and pollen AIT in children and adults.

\section{Introduction}

The prevalence of respiratory allergic diseases has been steadily increasing over the last decades in developed countries, affecting almost $20 \%$ of the world population. Allergic rhinitis (AR) and asthma frequently coexist in the same patient and represent a serious health problem. The link between AR and asthma was suggested many years ago, but was not properly considered as a unique airway disease until recently [1, $2 \bullet \bullet, 3]$. It is well known that AR in patients without asthma is a risk factor for asthma, especially in children [4], and more than $20 \%$ of patients with AR will develop asthma later in life. The European Community Respiratory Health Survey, including more than 6,000 patients, showed that the strongest risk for developing asthma occurred in those pa- tients with rhinitis and sensitization to several inhalant allergens, particularly house dust mites, but, even in the absence of atopy, rhinitis is a strong predictor of asthma [4].

Moreover, allergic sensitization tends to increase with age in atopic individuals, and the risk for developing new sensitizations goes with the risk for developing symptoms after exposure to these new sensitizers [5]. For all that reasons, treatment of respiratory allergic diseases is complex and includes allergen avoidance measures, pharmacologic treatment and allergen immunotherapy (AIT), when appropriate. Only specific measures related to allergen sensitization and AIT will be analyzed in this revision.

\section{Specific treatment options of respiratory allergic disease}

\section{Allergen avoidance measures}

The relationship between allergen exposure and respiratory symptoms is not always easy to recognize. With regard to seasonal allergens such as pollens, there is a direct relationship between high air pollen concentration and an increase in asthma-related and wheeze-related emergency room visits [6], as well as the opposite: patients allergic to pollen are asymptomatic outside the pollen season. In any case, avoidance of pollen grain exposure is not suitable in most cases.

In general, the relationship between allergen exposure and symptoms depends on the nature of the allergen and the size and aerodynamic characteristics of allergen-carrying particles [reviewed in $7 \bullet \bullet$ ].

For perennial allergens, such as cat and dog allergens, a close temporal relationship has been shown, and patients allergic to cat or dog dander react shortly after cat or dog exposure, respectively, so that the cause-effect relationship is obvious $[8,9]$. However, even if avoidance of pets is often possible, sometimes it becomes difficult due to usual behaviors of the 
community [10-12]. Concerning house dust mite (HDM), because of the larger size of HDM-carrying particles, a significant proportion of the allergen is deposited in the upper airways [13]. Exposure to high environmental levels of dust mite allergen is associated with an increased risk of sensitization,

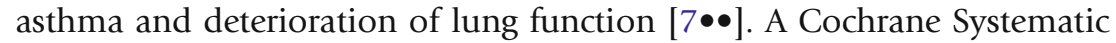
Review and meta-analysis did not detect specific clinical benefits from the use of both chemical and physical prophylactic environmental measures [14] in asthmatic patients sensitive to HDM, but trials included in the analysis tended to be small and of poor methodological quality. Probably, a combination of different methods is needed to achieve clinical improvement $[7 \bullet \bullet, 14,15 \bullet, 16]$. However, in general, physicians still go on recommending such measures as complementary treatment in respiratory allergy [17]. The role of allergen avoidance measures in the evolution of the respiratory disease is unknown.

\section{Allergen immunotherapy (AIT)}

The term 'allergen immunotherapy' (AIT) has been proposed to be universally used to refer to the class of therapies that aims at inducing immune tolerance to allergens [18]. Currently, two types of AIT are commonly used, depending on the route of allergen administration: subcutaneous (SCIT) and sublingual immunotherapy (SLIT). Both, SCIT and SLIT have shown to be

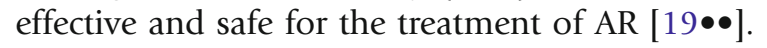

What guidelines for respiratory diseases recommend

1 The Global Initiative for Asthma (GINA) guidelines

The approach to allergic asthma that GINA (last version, December 2012 [20]) recommends is quite contradictory. On the one hand, GINA recognizes the role of allergens in asthma exacerbations, but on the other hand, the causative role of allergens in the development of asthma is questioned. This guideline considers the diagnosis of asthma in young children when a symptom pattern of recurrent wheeze/cough together with a positive family history and evidence of allergic sensitization is present, thus giving allergy a relevant role. The consideration of allergy as a systemic disease is beyond doubt. An example is the fact that after a single bronchial allergen challenge, there are increased levels of serum allergen-specific IgE for this allergen and enhanced Th2 response for up to 5 weeks after the challenge [21].

From a therapeutic point of view, GINA describes AIT as a modality of treatment with limited efficacy in adults with asthma [20]. However, results of successive Cochrane reviews, examining up to 88 randomized controlled clinical trials of SCIT compared to placebo on 3,459 patients with asthma, confirmed the efficacy of AIT not only in reducing asthma symptom scores (standardized mean difference [SMD] $-0.59 ; 95 \%$ CI, -0.83 to -0.35 ) and medication requirements (SMD, $-0.53 ; 95 \% \mathrm{CI},-0.80$ to -0.27 ) but also in achieving improvements of nonspecific and specific bronchial

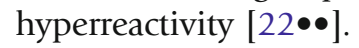

The long-term clinical effect of AIT and the potential of preventing development of asthma in children with allergic rhinitis are recognized in GINA, but due to potential systemic side effects, it is not recommended for general 
use [20]. The main problem is that the severe systemic reactions and deaths happened many years ago under uncontrolled circumstances [23, 24]. Nowadays, safety of AIT has been proven in both clinical trials and daily practice clinical settings $[25,26]$, but even with this available information, AIT is still not recommended for general use in this guideline. More contradictions appear when taking severity of the disease into account. On the one hand, AIT can be prescribed for well-controlled mild intermittent or mild persistent asthma, but on the other hand, GINA [20] remarks that AIT can be an option only if strict environmental avoidance and treatment with inhaled corticosteroids fail to control symptoms pointing at more severe forms of the disease. GINA insists on the lack of specific trials comparing AIT with pharmacologic treatment for asthma, but in fact, all well-designed placebo controlled studies performed with AIT measuring symptom and medication scores are equivalent for this purpose.

\section{Allergic Rhinitis and Its Impact on Asthma (ARIA) guidelines}

ARIA considers AIT for both adults and children patients with moderate to severe, persistent or intermittent AR $[1,2 \bullet \bullet, 3]$. The potential role of AIT in preventing asthma is specifically stated in this guideline, and recommendations about the management of AR and asthma when they coexist in the same patient are given.

According to ARIA, adults with AR but without asthma may use either SCIT or SLIT to treat pollen or HDM allergy. However, with regard to children, ARIA places a high value only on SCIT for reduction of symptoms and potential prevention of the development of asthma. Thus, SLIT may be recommended for adults with rhinitis caused by pollen or HDM, but only pollen SLIT should be recommended in children until the efficacy of HDM SLIT is proven in controlled clinical trials in children $[1,2 \bullet \bullet, 3]$. This different approach to SLIT depending on the allergen is difficult to understand. A systematic review of SLIT published in 2011 [27] revealed a significant improvement in symptoms (SMD, -0.49; $95 \%$ CI, -0.64 to -0.34 ) and medication scores (SMD, $-0.32 ; 95 \% \mathrm{CI},-0.43$ to -0.21) after analyzing 49 double-blinded, placebo-controlled, high quality studies. No differences were observed depending on the allergen or the age of patients (15 of these studies had been performed in children). Perhaps, the cautious recommendation given to children with HDM allergy is due to the small number of studies using this allergen.

A remarkable issue of ARIA is the consideration of AR and concomitant asthma as a unique airway disease. When treating patients (both children and adults) with AR and concomitant asthma, either SCIT or SLIT may be chosen, but there is higher quality evidence for effectiveness of the former than for the latter $[1,2 \bullet \bullet, 3]$.

As in GINA [20], the main limitation for using AIT in the treatment of AR is the risk of adverse side effects. However, many clinical trials and daily clinical practice rule out this supposedly high rate of serious side effects of AIT $[25,26]$.

\section{Global Allergy and Asthma European Network/European Academy of Allergy and Immunology $\left(\mathrm{GA}^{2} \mathrm{LEN} / \mathrm{EAACI}\right)$ guide for AIT}

This guideline offers a comprehensive set of recommendations on the use of AIT in the treatment of AR and asthma. Indications and contraindications for AIT are given [28]. 
For inhalant allergies, AIT may be of benefit in patients with proven IgE sensitization with clinical relevance. Therefore, it is indicated in IgE-mediated

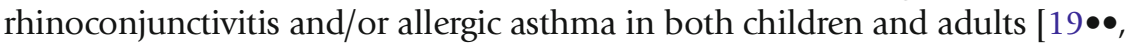
29-32]. For allergic rhinitis, AIT is appropriate whenever these patients require more than occasional use of oral or topical antihistamines, or intranasal corticosteroids for either repeated courses or continuous use. It is particularly useful in those patients who do not respond to current pharmacological treatment, or those who want to avoid chronic treatment and its undesirable side effects. As for allergic asthma, AIT is indicated in patients requiring more than modest consumption of $\beta 2$-agonists, or requiring inhaled corticosteroids; this includes patients with mild-to-moderate symptoms. In both cases, it is preferable to initiate AIT as soon as possible, before chronic irreversible changes occur. In summary, AIT is indicated in patients who have demonstrable evidence of specific IgE antibodies to clinically relevant allergens and whose symptoms are

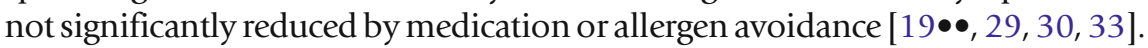

Contraindications in AIT, according to the last EAACI position statement, are divided into absolute and relative [30]. Serious immunological diseases, major cardiovascular disease, cancer, chronic infections, uncontrolled severe asthma, treatment with $\beta$-blockers, lack of compliance and severe psychological disorders are absolute contraindications. Pregnancy, severe atopic eczema, elderly and patients below 5 year of age are relative contraindications. Under a critical point of view, it can be argued how evidence based these contraindications are. Some of these recommendations are based upon a theoretical interaction between autoimmune disorders and the immunological effect of AIT, but little or no solid evidence concerning this interaction has been ever reported. Others, such as $\beta$-blockers or cardiovascular diseases, were established because such conditions might makes the treatment of an eventual systemic reaction due to AIT difficult.

\section{Are guidelines accurately followed by physicians?}

Two different studies have addressed this question [34, 35]. The first evaluation [34] is an observational multicenter study that was carried out in 518 patients recruited from 34 Allergy Departments in Italy, trying to find out if prescriptions of AIT followed recommendations of ARIA and GINA guidelines. A questionnaire shared by patients and physicians was filled in by all consecutive mite-allergic patients aged 14 and older, attending a specialist unit. AR and asthma severity were assessed using ARIA and GINA guidelines classification $[1,20]$, and patients were classified according to the level of severity for both entities. A significant relationship between age and prescription of AIT was seen (the probability of AIT prescription is between 4.5 and 6 times higher in teenagers). Another independent factor was the tendency to worsening of the disease, as evaluated by the doctor (odd ratio 2.5, $95 \% \mathrm{CI}, 1.4-4.5$ ). Taken into account that patients are referred to the specialist based on the severity of the disease, a discrepancy was seen between ARIA and GINA, because GINA discards AIT in the most severe forms of the disease [20]. The most common condition for AIT prescription in this survey (56\%) was persistent AR with coexisting, intermittent asthma [34].

Meanwhile, a cross-sectional survey performed in France showed that more than $50 \%$ of general practitioners and ear-nose-throat (ENT) 
physicians who had admitted to knowing the ARIA guidelines did not provide a different treatment strategy, including AIT, compared to those who did not manage ARIA [35].

\section{Long-term efficacy of AIT: definition}

Depending on the duration of the studies, the efficacy of AIT can be divided into short-term, sustained effect and long-term. We will refer to long-term efficacy as "the prolonged clinically relevant benefit that persists after stopping the treatment" [36].

In 1988, Mosbech and Østerballe first demonstrated that the effect of AIT could last after termination of treatment [37]. Thirty-eight patients (aged 1543 years) who had participated in a controlled study of grass pollen SCIT for 2 years and 4 months from 1978 to 1980 were followed up in 1986, 6 years after stopping the treatment. Symptom and medication scores were prospectively recorded during the pollen season in 1986 using the same cards they had filled in during the clinical trial. The median symptoms for the sixth season were more than $33 \%$ of the pretreatment values in spite of a $10 \%$ higher pollen exposure.

Durham et al. [38] have shown "long-term clinical efficacy" after providing a prolonged clinical remission of symptoms for at least 3 years following discontinuation of SCIT with a grass-pollen extract. In this study, 32 patients who had received grass pollen SCIT for 3 or 4 years were randomized to either continued maintenance SCIT with the same vaccine or placebo injections for one more year. Fifteen matched controls with no previous contact with AIT were used as controls. Authors found no differences between patients from the placebo or the active treatment group, meaning that no additional benefit is obtained after one more year of treatment. On the contrary, patients who had never received AIT had significantly higher symptom and medication scores than those with previous AIT. It seems that no recrudescence of symptoms would occur when therapy was discontinued after successful treatment.

As the long-term efficacy definition implies, to find out if there is any clinical benefit from AIT after treatment is stopped, we selected and reviewed clinical trials conducted to evaluate the persistence or not of clinical efficacy once treatment has been discontinued.

\section{Evidence for long-term efficacy with pollen SCIT}

The Preventive Allergy Treatment (PAT) study is a European multi-center study designed to find out if 3 years of SCIT could prevent children from developing asthma. Two hundred and five children ( 6 to 14 years) suffering from seasonal AR due to birch and/or grass pollen were recruited. Before baseline monitoring, 42 of them were classified as having mild intermittent asthma with no need of daily medication. Patients were randomized either to receive SCIT with standardized allergen extracts of grass pollen and/or birch pollen or to an open control group. Finally, 79 patients with no previous asthma and 18 with mild intermittent asthma were allocated in the active group, whereas 72 and 22, respectively, went to the control group with no 
AIT. After 3 years of treatment, a significantly higher number of children in the control group developed asthma (odds ratio of 2.52, $95 \% \mathrm{CI}, 1.3$ to $5.1 ; P<0.05$, favoring the hypothesis that SCIT can prevent the development of asthma) [39-41]. Two years and 7 years after termination of the treatment (5-year and 10-year follow-up), the preventive effect of SCIT persisted and was even greater, reaching an odds ratio of 4.6 (95\% CI, 1.5 to 13.7) in the latter analysis of the 117 available patients without asthma before the start of the study [40, 41].

Despite not being double-blinded or placebo-controlled, the PAT study is a model mentioned worldwide to support the long-term preventive effect of immunotherapy. Authors defended their decision not to include subcutaneous placebo treatment for ethical reasons due to the length of the study. They have used the same methodology for all follow-up revisions, but the number of patients is small.

A similar approach was made by Eng et al. using a grass-pollen allergoid and proving a similar benefit 12 years after discontinuation of SCIT in a small group of 14 children compared to a similar group of non-treated grass-allergic children [42, 43].

As it has been stated in the definition of "long-term efficacy", studies from Mosbech and Østerballe in 1988 [37] and Durham et al. in 1999 [38] both demonstrate that clinical improvement in symptoms and need-of-rescue medication scores after grass pollen AIT persist once treatment is stopped. However, Naclerio et al. [44], using a similar scheme of treatment, failed to show this long-lasting effect for ragweed SCIT. This study involved 20 adult patients (aged 18 to 55 years) with AR and mild asthma who had received ragweed AIT for at least 3 years. After this period of time, patients were double-blinded and randomized to either receive one more year of ragweed or placebo injections. One year after discontinuing successful ragweed AIT, authors found contrasting results. On the one hand, nasal provocation test results were similar to baseline in the placebo group. On the other hand, symptom and medication scores remained decreased in this group, suggesting a clinically prolonged effect of previous AIT. Nevertheless, the small number of patients limits the value of the results.

In 2005, Corrigan et al. [45] published the results of a multi-center, double-blinded, placebo-controlled study of a high-dose hypoallergenic grass pollen extract in adults. One hundred and fifty-four patients (aged 1860 years) allergic to grass pollen with AR and with or without mild asthma were enrolled in the study. Patients were divided into two groups (77 active / 77 placebo) and received at least nine injections preseasonally for two consecutive years. Clinical efficacy assessed by symptoms and medication scores, visual analogue scales and quality of life questionnaires showed efficacy after the first and second years of treatment. Once finished the clinical trial, all patients (active and placebo groups) received one year of SCIT [45]. A 3-year follow-up study performed in a sub-group of 26 of those patients who had previously received three courses of treatment from two centers in Germany were compared to a group of grass allergic patients who had never received AIT before. Symptom-medication score was significantly reduced 
and quality of life significantly improved in the ex-SCIT group, supporting the long-term effect of SCIT in adults [46].

\section{Evidence for long-term efficacy with mites SCIT}

\section{Children and adults}

Few studies have addressed this issue. Due to ethical or methodological reasons, no double blind studies have been performed following patients after stopping the treatment with mite SCIT. In an open study performed by Des Roches et al. [47] in both child and adult asthmatic patients treated with a standardized

Dermatophagoides pteronyssinus extract, it was shown that less than 3 years of treatment is associated with a relapse of asthma symptoms within the first years after discontinuation. In a retrospective survey [48], 48 asthmatic adults allergic to HDM or to both HDM and grass pollen who had received SCIT during childhood were compared to 42 asthmatic adults who has been treated with conventional treatment. For that purpose, patients and controls were assessed by standardized questionnaire and lung function tests. Even though it is not a prospective blind study, it resembles clinical practice, and it is noteworthy that 9 years after finishing SCIT, asthma symptoms were significantly reduced in the AIT group. Along this line, Stelmach et al. [49] compared the duration of the effect after 3-year and 5-year SCIT with HDM. The study was carried out in 90 children suffering from persistent allergic asthma and requiring inhaled corticosteroids at a minimum dose between 400 and $800 \mu \mathrm{g}$ of budesonide before initiating SCIT (in the active group) or before entering the study (control group). The study had a retrospective phase, consisting of collecting data from patients who had received HDM SCIT for 3 or 5 years (30 patients in each group) and from a control group with no AIT, but wellestablished budesonide needs for the previous 2 years. During a prospective follow-up of 3 years, symptom and medication scores were recorded, and a significant improvement was evident in both SCIT groups with no need of inhaled corticosteroids in contrast to the control group, which had maintained a similar dose. These results were in agreement with those previously published showing than HDM SCIT administered during 3 years was enough to reach a significant improvement in asthma symptoms [50]. The study was performed in both children and adults, and no detailed information on the number of children or specific results for this age group is detailed. Authors showed a small margin of benefit after 2 more years of treatment for AR symptoms.

\section{Evidence for long-term efficacy with pollen SLIT}

\section{Children}

Following the requirements of the Pediatric Committee of the EMA (PDCO) [51] for the development of new allergen products as registered tablets in the pharmaceutical market, more studies will be available to show this long-term effect. The Pediatric Investigation Plan (PIP) of the European Medicine Agency (EMA) was born to support the idea that AIT should only be given to children if a disease-modifying effect is demonstrated after 3 years of treatment, followed by 2 more years without it. In this context, a European multi-centric 
initiative aimed to investigate the preventive effect of marketed grass tablets (SLIT), the so-called GAP study, [52] is being carried out under a 5-year-term double-blind, placebo-controlled, designed study, and solid evidence on the preventive effect of AIT for developing asthma in children suffering AR is expected from it, but the study is still underway.

Adults

Long-term clinical efficacy in grass pollen-induced rhinoconjunctivitis has been demonstrated in at least two independent well-designed clinical trials of grass pollen tablets $[53 \bullet \bullet, 54 \bullet \bullet]$ and one clinical trial using dual grass pollen and HDM SLIT drops [55].

A randomized, multi-centre, double-blind, placebo-controlled

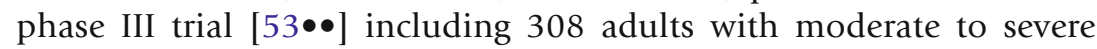
grass pollen AR who had completed a 3-year course of perennial SLIT with a tablet of grass pollen $(n=170)$ or placebo $(n=138)$ was extended to cover 2 years of follow-up without treatment. During follow-up, the mean average daily symptoms were reduced by $26 \%$ in the active group ( $P=0.0007$ compared to placebo), and a reduction of medication consumption of $29 \%$ was evident in the active group $(P=0.0215)$. The level of efficacy was similar to that obtained during the third year of treatment. Similar results were obtained with a five-grass pollen sublingual tablet administered pre-seasonally and co-seasonally during 3 years in a multi-centre, randomized, double-blind, placebo-controlled study including 457 patients (294/active and 163/placebo), supporting this long-term effect [54••]. Finally, Swamy et al. [55] found significant improvements in symptom and medication scores 1 year after stopping a 1-year course of SLIT with dual HDM and grass pollen. This is a randomized, double-blind, placebo-controlled, phase I study performed in one centre in both children and adults. Patients were allocated to receive either active treatment $(n=20)$ or placebo $(n=10)$. One year after stopping dual SLIT, significant decreased symptoms and medication scores were recorded.

\section{Evidence for long-term efficacy with mites SLIT}

\section{Children}

In a prospectively designed, open, parallel-group controlled study, Di Renzo et al. [56] treated children with HDM-allergic asthma for 4 to 5 years with either HDM-SLIT plus pharmacological treatment $(n=35)$ or pharmacological treatment alone (control group, $n=25)$. Patients were evaluated at baseline, at the end of the treatment period and 4-5 years after discontinuation. Clinical evaluation (symptom and medication scores) at baseline and 5 and 10 years later showed a significant improvement in those patients who had received SLIT $(P<0.001)$, but not in the control group. A similar open study was performed in children with HDM SLIT, showing a 
steroid-sparing effect that persists for at least 6 months [57] after discontinuation of SLIT in the active group.

The main limitation of these studies is that they are open, but double-blind studies for such a long period of follow-up are not feasible for ethical and practical reasons. However, a double-blind design for the randomization and during the treatment period with an open follow-up at the end of the treatment would be desirable.

A prospective, open, controlled, 4-parallel-group partially randomized study involving adults patients (aged 18-65 years) with AR and mild intermittent asthma due to HDM allergy was designed to evaluate the long-term effects of HDM SLIT according to the duration of the treatment [58]. Patients were placed into an active group receiving a glycerinated solution of HDM plus drugs or in a control group treated with drugs only. Assignment was made according to patients` preference. From a previous sample of 198 patients who met inclusion criteria, 78 entered the study. Symptom and medication scores were recorded from September to February, and lung function tests were performed yearly at the end of the winter season. Patients under SLIT were randomized to receive treatment for 3, 4 or 5 years. After 15 years of observation, 19 patients dropped out because of protocol deviations. No differences were seen among patients treated for 4 or 5 years, but there was a significant improvement in those treated 4 or 5 years with respect to those who had been treated for 3 years. Up to 7 years after discontinuation of HDM SLIT, patients actively treated registered better symptom and medication scores and better lung function tests than those with drugs only. These promising results have limitations, because of the lack of an initial proper randomization and the absence of a placebo group. Moreover, the high rate of dropouts, many of them in the control group, weakens the value of the results.

\section{Compliance with Ethics Guidelines}

\section{Conflict of Interest}

Carmen Vidal received honoraria from ALK-Abello Laboriatory and Stallergenes Laboratory and had travel/ accommodations expenses covered or reimbursed by EAACI.

Pablo Rodríguez del Río had travel/accommodations expenses covered or reimbursed by EAACI.

Ana Tabar received honoraria from ALK-Abello Laboriatory and Stallergenes Laboratory.

Carmen Moreno received honoraria from ALK-Abello Laboratory and had travel/accommodations expenses covered or reimbursed by EAACI.

\section{Human and Animal Rights and Informed Consent}

This article does not contain any studies with human or animal subjects performed by any of the authors. 


\section{References and Recommended Reading}

Papers of particular interest, published recently, have been highlighted as:

- Of importance

- Of major importance

1. Bousquet J, Van Cauwenberge P, Khaltaev N, Aria Workshop Group; World Health Organization. Allergic rhinitis and its impact on asthma. J Allergy Clin Immunol. 2001;108:S147-334.

2.• Brozek JL, Bousquet J, Baena-Cagnani CE, et al. Allergic rhinitis and its impact on asthma (ARIA) guidelines: 2010 Revision. J Allergy Clin Immunol. 2010;126:466-77.

More recent revision of this guideline first published in 2001, trying to unify criteria for classifying patients and their treatment options.

3. Bousquet J, Scünermann HJ, Samolinski B, et al. Allergic rhinitis and its impact on asthma (ARIA): achievements in 10 years and future needs. J Allergy Clin Immunol. 2012;130:1049-62.

4. Shaaban R, Zureik M, Soussan D, et al. Rhinitis and onset of asthma: a longitudinal population-based study. Lancet. 2008;372:1049-57.

5. Sun HL, Yeh CJ, Ku MS, et al. Coexistence of allergic diseases: patterns and frequencies. Allergy Asthma Proc. 2012;33:e1-4.

6. Darrow LA, Hess J, Rogers CA, et al. Ambient pollen concentrations and emergency department visits for asthma and wheeze. J Allergy Clin Immunol. 2012;130:630-8.

7.• Custovic A, Simpson A. The role of inhalant allergens in allergic airway disease. J Investig Allergol Clin Immunol. 2012;22:393-401.

Excellent review of the role of allergens in allergic respiratory disease with a detailed description of avoidance measures and their utility.

8. Custovic A, Murray CS, Gore RB, et al. Controlling indoor allergens. Ann Allergy Asthma Immunol. 2002;88:432-41.

9. Custovic A, Simpson B, Simpson A, et al. Relationship between mite, cat, and dog allergens in reservoir dust and ambient air. Allergy. 1999;54:612-6.

10. Bertelsen RJ, Instanes C, Granum B, et al. Gender differences in indoor allergen exposure and association with current rhinitis. Clin Exp Allergy. 2010;40:1388-97.

11. Morris DO. Human allergy to environmental pet danders: a public health perspective. Vet Dermatol. 2010;21:441-9.

12. Abramson SL, Turner-Henson A, Anderson L, et al. Allergens in school settings: results of environmental assessments in 3 city school systems. J Sch Health. 2006;76:246-9.

13. De Blay F, Heymann PW, Chapman MD, et al. Airborne dust mite allergens: comparison of group II allergens with group I mite allergen and catallergen Fel d I. J Allergy Clin Immunol. 1991;88:919-26.

14. Nurmatov U, van Schayck CP, Hurwitz B, et al. House dust mite avoidance measures for perennial allergic rhinitis: an updated Cochrane systematic review. Allergy. 2012;67:158-65.

15. Gehring U, de Jongste JC, Kerkhof M, et al. The 8-year follow-up of the PIAMA intervention study assessing the effect of mite-impermeable mattress covers. Allergy. 2012;67:248-56.

Prospective study aimed at investigating the preventive effect of using mite-impermeable mattress covers in high-risk children for allergy. Even though this intervention was useful to reduce mite exposure, it failed to protect children from allergic sensitization.

16. Matsui EC. Environmental control for asthma: recent evidence. Curr Opin Allergy Clin Immunol. 2013;13:417-25.

17. Pingitore G, Pinter E. Environmental interventions for mite-induced asthma: a journey between systematic reviews, contrasting evidence and clinical practice. Eur Ann Allergy Clin Immunol. 2013;45:74-7.

18. Calderón MA, Casale T, Cox L, et al. Allergen immunotherapy: a new semantic framework from the European Academy of Allergy and Clinical Immunology/American Academy of Allergy, Asthma and Immunology/PRACTALL consensus report. Allergy. 2013;68:825-8.

19.• Burks AW, Calderon MA, Casale T, et al. Update on allergy immunotherapy: American Academy of Allergy, Asthma and Immunology/European Academy of Allergy and Clinical Immunology/PRACTALL consensus report. J Allergy Clin Immunol. 2013;131(5):1288-96.

Consensus report reviewing definition of allergy immunotherapy, its mechanism of action and guidelines for clinical practice.

20. Global Strategy for Asthma Management and Prevention. Global Initiative for Asthma (GINA) 2011. http://www.ginasthma.org. 
21. van de Pol MA, Lutter R, van Ree R, et al. Increase in allergen-specific IgE and ex vivo Th2 responses after a single bronchial challenge with house dust mite in allergic asthmatics. Allergy. 2012;67:67-73.

22.• Abramson MJ, Puy RM, Weiner JM. Injection allergen immunotherapy for asthma. Cochrane Database Syst Rev. 2010;4(8):CD001186

Meta-analysis proving the efficacy of subcutaneous immunotherapy in improving asthma symptoms, medication scores and both specific and unspecific bronchial hyperreactivity.

23. CSM Update: desensitizing vaccines. Br Med J (Clin Res Ed) 1986;293:948-53.

24. Bernstein DI, Wanner M, Borish L, et al. Twelve-year survey of fatal reactions to allergen injections and skin testing: 1990-2001. J Allergy Clin Immunol. 2004;113:1129-36.

25. Moreno C, Cuesta-Herranz J, Fernández-Távora L, et al. Immunotherapy safety: a prospective multicenter monitoring study of biologically standardized therapeutic vaccines for allergic diseases. Clin Exp Allergy. 2004;34:527-31.

26. Fernández-Távora L, Justicia JL, Moreno C, et al. Safety evaluation of rapid build-up schedules with IR-standardized allergen extracts for subcutaneous immunotherapy of allergic respiratory diseases. Expert Opin Drug Saf. 2011;10:947-55.

27. Radulovic S, Wilson D, Calderon M, et al. Systematic reviews of sublingual immunotherapy (SLIT). Allergy. 2011;66:740-52.

28. Zuberbier T, Bachert C, Bousquet J, et al. GA2LEN/ EAACI pocket guide for allergen-specific immunotherapy for allergic rhinitis and asthma. Allergy. 2010;65:1525-30.

29. Bousquet J, Demoly P, Michael FB. Specific immunotherapy in rhinitis and asthma. Ann Allergy Asthma Immunol. 2001;87(Suppl):38-42.

30. Alvarez-Cuesta E, Bousquet J, Canonica GW, et al. Standards for practical allergen-specific immunotherapy. Allergy. 2006;61(Suppl):1-20.

31. Passalacqua G, Canonica GW. Specific immunotherapy in asthma: efficacy and safety. Clin Exp Allergy. 2011;41:1247-55.

32. Calderon MA, van Wijk RG, Eichler I, et al. Perspectives on allergen-specific immunotherapy in childhood: An EAACI position statement. Pediatr Allergy Immunol. 2012;23:300-6.

33. DuBuske L. Appropriate and inappropriate use of immunotherapy. Ann Allergy Asthma Immunol. 2001;87(Suppl):56-67.

34. Antonicelli L, Braschi MC, Biló B, et al. Congruence between international guidelines and mite specific immunotherapy prescribing practices. Respir Med. 2011;105:1441-8.

35. Demoly P, Concas V, Urbinelli R, et al. Spreading and impact of the World Health Organization`s allergic rhinitis and its impact on asthma guidelines in everyday medical practice in France. Ernani survey. Clin Exp Allergy. 2008;38:1803-7.

36. European Medicines Agency. Committee for Medicinal Products for Human Use (CHMP). Guideline of the clinical development of products for specific immunotherapy for the treatment of allergic diseases. 2008 EMEA/CHMP/EWP/18504/2006.

37. Mosbech H, Østerballe O. Does the effect of immunotherapy last after termination of treatment? Follow-up study in patients with grass pollen rhinitis. Allergy. 1989;43:523-9.

38. Durham SR, Walker SM, Varga EM, et al. Long-term clinical efficacy of grass-pollen immunotherapy. $\mathrm{N}$ Eng J Med. 1999;341:468-75.

39. Möller C, Dreborg S, Ferdousi HA, et al. Pollen immunotherapy reduces the development of asthma in children with seasonal rhinoconjunctivitis (the PAT-study). J Allergy Clin Immunol. 2002;109:251-6.

40. Niggeman B, Jacobsen L, Dreborg S, et al. Five-year follow up on the PAT study: Specific immunotherapy and long-term prevention of asthma in children. Allergy. 2006;61:855-9.

41. Jacobsen L, Niggeman B, Dreborg S, et al. Specific immunotherapy has long-term preventive effect of seasonal and perennial asthma: 10-year follow-up on the PAT study. Allergy. 2007;62:943-8.

42. Eng PA, Reinhold M, Gnehm HPE. Long-term efficacy of preseasonal grass pollen immunotherapy in children. Allergy. 2002;57:306-12.

43. Eng PA, Borer-Reinhold M, Heijnen IAF, et al. Twelve-year follow-up after discontinuation of preseasonal grass pollen immunotherapy in childhood. Allergy. 2006;61:198-201.

44. Naclerio RM, Proud D, Moylan B, et al. A doubleblind study of the discontinuation of ragweed immunotherapy. J Allergy Clin Immunol. 1997;100:293-300.

45. Corrigan CJ, Kettner J, Doemer C, et al. Efficacy and safety of preseasonal-specific immunotherapy with an aluminium-adsorbed six-grass pollen allergoid. Allergy. 2005;60:801-7.

46. Dominicus R. 3-year`s long-term effect of subcutaneous immunotherapy (SCIT) with high-dose hypoallergenic 6-grass pollen preparation in adults. Eur Ann Allergy Clin Immunol. 2012;44:135-40.

47. Des Roches A, Paradis L, Knani J, et al. Immunotherapy with a standardized Dermatophagoides pteronyssinus extract. V. Duration of efficacy of immunotherapy after its cessation. Allergy. 1996;51:430-3.

48. Cools M, Van Bever HP, Weyler JJ, et al. Long-term effects of specific immunotherapy, administered during childhood, in asthmatic patients allergic to either house-dust mite or to both house-dust mite and grass pollen. Allergy. 2000;55:69-73. 
49. Stelmach I, Sobocinska A, Majak P, et al. Comparison of the long-term efficacy of 3- and 5-year house dust mite allergen immunotherapy. Ann Allergy Asthma Immunol. 2012;109:274-8.

50. Tabar AI, Arroabarren E, Echechipia S, et al. Three years of specific immunotherapy may be sufficient in house dust mite respiratory allergy. J Allergy Clin Immunol. 2011;127:57-63.

51. The European Parliament Council. Regulation (EC) No 1901/2006 on medicinal products for paediatric use and amending Regulation (EEC) No 1768/92, Directive 2001/20/EC, Directive 2001/83/EC and Regulation (EC) No 726/2004. OJ 2006;378:1-19.

52. Valovirta E, Berstad AK, de Blic J, et al. Design and recruitment for the GAP trial, investigating the preventive effect on asthma development of an SQstandardized grass allergy immunotherapy tablet in children with grass pollen-induced allergic rhinoconjunctivitis. Clin Ther. 2011;33:1537-46.

53.• Durham SR, Emminger W, Kapp A, et al. SQ-standardized sublingual grass immunotherapy: Confirmation of disease modification 2 years after 3 years of treatment in a randomized trial. J Allergy Clin Immunol. 2012;129:717-25.

One of the most recent studies proven long-term efficacy of tablet sublingual immunotherapy.
54.• Didier A, Malling HJ, Worm M, et al. Post-treatment efficacy of discontinuous treatment with 300IR 5-grass pollen sublingual tablet in adults with grass pollen-induced allergic rhinoconjunctivitis. Clin Exp Allergy. 2013;43:568-77.

One of the most recent studies proven long-term efficacy of tablet sublingual immunotherapy.

55. Swamy RS, Reshamwala N, Hunter T, et al. Epigenetic modifications and improved regulatory T-cell function in subjects undergoing dual sublingual immunotherapy. J Allergy Clin Immunol. 2012;130:215-24.

56. Di Rienzo V, Marcucci F, Puccinelli P, et al. Longlasting effect of sublingual immunotherapy in children with asthma due to house dust mite: a 10-year prospective study. Clin Exp Allergy. 2003;33:206-10.

57. Ozdemir C, Yazi D, Gocmen I, et al. Efficacy of longterm sublingual immunotherapy as an adjunct to pharmacotherapy in house dust mite-allergic children with asthma. Pediatr Allergy Immunol. 2007; 18:508-15.

58. Marogna M, Spadolini I, Massolo A, et al. Long-lasting effect of sublingual immunotherapy according to its duration: a 15-year prospective study. J Allergy Clin Immunol. 2011;126:969-75. 